\title{
Exploring the Less Common Pathogens of Infectious Diarrhea
}

\author{
Steven Toffel ${ }^{1}$, Lymaries Velez ${ }^{1}$, Elizabeth Tremblay ${ }^{2}$, Kairav Shah $^{3}$, Kenneth Rand ${ }^{4}$, Jena Auerbach ${ }^{5}$, \\ Kathryn Anne Potter ${ }^{5}$, Stacy G. Beal ${ }^{4}$ \\ ${ }^{1}$ University of Florida College of Medicine; \\ ${ }^{2}$ UF Health Shands Hospital, Department of Infection Control \\ ${ }^{3}$ University of Florida, Department of Internal Medicine, Division of Infectious Diseases and Global Medicine \\ ${ }^{4}$ University of Florida, Department of Pathology \\ ${ }^{5}$ University of Florida, Department of Dermatology
}

\begin{abstract}
Objective: Gastrointestinal (GI) panels have allowed for faster and accurate detection, treatment, and control of pathogens. Because of the large number of potential pathogens included in the panel the clinical significance and manifestations of some organisms, such as Enteroaggregative Escherichia coli (EAEC) and Enteropathogenic E. coli (EPEC), remains undetermined.

Methodology: We performed a single-institution retrospective chart review for 222 patients with a stool sample tested on BioFire FilmArray Gastrointestinal Panel (GI Panel) between June 1, 2016 and March 9, 2017.

Results: Of the 222 patients, four had EAEC and 17 and EPEC. Patients who tested positive for EAEC and EPEC were younger (26.3 years and 33.9 years, respectively) than patients that did not test positive for a GI pathogen (47.7 years). In cases where multiple organisms were detected, EPEC was present $56.3 \%$ of the time. Analysis of symptoms showed that EPEC patients had a high prevalence of abdominal pain $(p=0.0425)$ and vomiting $(p=0.0045)$, but not diarrhea, when compared to the presence of these symptoms in patients with no targets detected. With only four patients positive for EAEC in this study, the results of symptoms in EAEC patients were inconclusive. Cases involving EPEC and EAEC were treated similarly to other cases of diarrhea with no significant difference in the number of imaging studies and medication changes.
\end{abstract}

Conclusions: While further studies are required to determine the specific clinical significance of EAEC or EPEC, it is evident that patients positive for EPEC often experience abdominal pain and diarrhea, suggesting EPEC may be more pathogenic than previously thought. J Microbiol Infect Dis 2019; 9(1):1-9.

Keywords: Diarrhea, Gastrointestinal Panel, Escherichia coli

\section{INTRODUCTION}

Acute infectious diarrhea is a leading cause of outpatient visits and hospitalizations in the United States, affecting 179 million people annually $[1,2]$. Globally, it is estimated that there are 1.7 billion cases of diarrhea disease every year [3].

The burden of diarrhea is largest in children under the age of 5 , where diarrhea is the second leading cause of death, killing 760,000 children every year [3,4]. Many of these diarrheal-related deaths occur in developing countries where water sanitation and sewage disposal are lacking.
Widespread diarrhea infectious in developed countries can ultimately occur by unsafe food and water distribution [4]. Many approaches have been taken to help prevent this problem, such as the development of the Foodborne Diseases Active Surveillance Network (FoodNet) which actively monitors the incidence of laboratory-confirmed infections of several pathogens [5].

Because the cause of acute infectious diarrhea is rarely directly identified based solely on clinical presentation, the use of laboratory tests plays a significant role in the immediate diagnosis of acute diarrhea [6]. It has been proven that correctly identifying the etiology of 
acute infectious diarrhea can have a significant impact on containment of the disease as well as clinical care, as treatment can be delivered earlier and shorten the duration of symptoms $[7,8]$.

While the use of conventional methods such as stool cultures and enzyme immunoassays (EIA) have played a significant role, there has been increased interest in the use of molecular diagnostic tests such as multiplex polymerase chain reaction (PCR) because of their ability to accurately and rapidly identify pathogens. One such assay is the BioFire FilmArray ${ }^{\circledR}$ Gastrointestinal (GI) Panel, which identifies 22 gastrointestinal pathogens in under an hour from a single stool sample (See Table 1) [9]. Other studies have shown that this test performs with sensitivity and specificity $>90 \% \quad[9,10]$. Enteroaggregative and Enteropathogenic Escherichia coli can be identified on the Gl Panel, but conventional methods do not routinely identify these organisms. Therefore, the clinical significance and manifestations are unclear. Are these organisms the cause of disease or innocent bystanders?

While numerous studies have examined the GI Panel results, there has not been a study focusing on the impact EAEC and EPEC infections have had on the patient clinically. Prior studies have showed that both EAEC and EPEC have the potential to elicit an inflammatory process and diarrhea [11,12], but with many non-disease causing subtypes of both EAEC and EPEC, detection of the organism does not equate to illness.

These two pathotypes of $E$. coli are also referred to as diarrheagenic E. coli as they are both associated with diarrhea. Enteroaggregative $E$. coli (EAEC) is a pathotype known for the ability to aggregate with each other and epithelial cells, particularly producing a "stacked-brick" appearance when grown with HEp-2 epithelial cells [13-15]. In addition to its adherence properties, its production of toxins results in mucosal inflammation of the gut [11]. The strains of EAEC are heterogenous, varying in colonization, virulence factor-encoding genes, and the host response it can elicit [13]. With its varied pathogenicity, EAEC has been linked to numerous infectious diarrhea outbreaks around the world [16-18]. Numerous PCR assays have been developed to identify EAEC presenting the aggR gene, a virulence gene strongly associated with pathogenic strains of EAEC $[19,20]$. Nonetheless, there are strains of EAEC containing agg $R$ that do not elicit complications such as diarrhea, as well as pathogenic strains of EAEC that do not contain the aggR gene. These atypical strains thus present a challenge for the proper identification of pathogenic strains of EAEC. Additionally, identification of the aggR gene by PCR systems does not necessarily equate to a complication of diarrhea.

Table 1. BioFire FilmArray GI Panel Organisms detected.

\begin{tabular}{l}
\hline Bacteria \\
Campylobacter (jejuni, coli and upsaliensis) \\
Clostridium difficile (toxin A/B) \\
Plesiomonas shigelloides \\
Salmonella \\
Yersinia enterocolitica \\
Vibrio (parahaemolyticus, vulnificus and cholerae) \\
Vibrio cholerae \\
\hline Diarrheagenic E. coli/Shigella \\
Enteroaggregative E. coli (EAEC) \\
Enteropathogenic E. coli (EPEC) \\
Enterotoxigenic E. coli (ETEC) It/st \\
Shiga-like toxin-producing E. coli (STEC) stx1/stx2 \\
E. coli O157 \\
Shigella/Enteroinvasive E. coli (EIEC) \\
\hline Parasites \\
Cryptosporidium \\
Cyclospora cayetanensis \\
Entamoeba histolytica \\
Giardia lamblia \\
Viruses \\
Adenovirus F 40/41 \\
Astrovirus \\
Norovirus Gl/GII \\
Rotavirus A \\
Sapovirus (I, II, IV and V) \\
\end{tabular}

Enteropathogenic E. coli (EPEC) is a pathotype that induces an attaching and effacing $(A / E)$ lesion, forming a unique pedestal-like structure on intestinal epithelial cells $[12,21,22]$. The interactions EPEC has with host intestinal epithelial cells disrupts water and solute transport leading to watery diarrhea, which is 
especially common in children $[11,12]$. EPEC is often divided into typical EPEC (tEPEC) and atypical EPEC (aEPEC) based on the presence of EPEC adherence factor plasmid ( $p E A F$ ) in tEPEC involved in colonization [21,23]. The specific role of tEPEC is often debated as aEPEC serotypes have also been linked to diarrheal outbreaks around the world [21]. Additionally, numerous epidemiological studies are suggesting aEPEC is not only more prevalent but more important to monitor in diarrheal outbreaks [24-31]. In previous studies using the BioFire FilmArray ${ }^{\circledR}$ GI Panel, EPEC was found in $37.1 \%$ of samples collected from children between age 1 and 5 with diarrhea. With how prevalent EPEC is in samples, we must question the significance these bacteria have in acute infectious diarrhea [9].

Overall, it is evident that EAEC and EPEC both have the capacity to elicit a diarrheal infection, perhaps some strains more than others. In this study, we aimed to understand the clinical significance and manifestation of Enteroaggregative and Enteropathogenic Escherichia coli found in patients undergoing diagnostic testing for gastroenteritis.

\section{METHODS}

\section{Study Design and Setting}

This study is a retrospective review conducted at UF Health Shands Hospital in Gainesville, Florida following approval University of Florida's Institutional Review Board.

\section{Selection of patient population}

All patients who had the FilmArray Gl Panel testing between June 1, 2016 and March 9, 2017 at UF Health Shands Hospital were considered for this study. At this time, the laboratory was performing a clinical evaluation of the assay by performing it on samples collected for a stool culture. The providers ordered stool cultures, and in addition to performing the stool culture, we also tested the same sample on the Gl Panel.

\section{Methods and measures}

All records from a patient's hospital admission were reviewed. Demographic data including age, gender and BMI were collected. Additionally, information on the patient's vitals, co-morbidities, symptoms, CBC and basic metabolic panels, imaging studies, recent antibiotic use, recent travel out of the country, and GI Panel results were also documented.

Study data were collected and managed using REDCap electronic data capture tools hosted at University of Florida. [32] REDCap (Research Electronic Data Capture) is a secure, web-based application designed to support data capture for research studies, providing: 1) an intuitive interface for validated data entry; 2) audit trails for tracking data manipulation and export procedures; 3) automated export procedures for seamless data downloads to common statistical packages; and 4) procedures for importing data from external sources.

\section{Statistical analysis}

Statistical analysis was conducted using Excel. "N-1" Chi-squared test, as recommended by Campbell (2007) and Richardson (2011) [33,34], was performed to evaluate the prevalence of symptoms in patients with specific organisms in comparison to the prevalence of symptoms with no targets detected.

\section{RESULTS}

\section{FilmArray GI Panel Findings}

This study identified 222 patients who were evaluated via FilmArray Gl Panel between June 1, 2016 and March 9, 2017. Patients >18 years old were considered to be in the adult population in this study (175 total) while those from patients under $\leq 18$ years old and were considered to be in the pediatric (47 total) age group. The FilmArray Gl Panel detected at least one organism, in 61 of the 222 specimens analyzed $(27.5 \%)$. Overall, the most prevalent organism in adults detected by the FilmArray GI Panel were Norovirus and EPEC, which were detected in $29 \%$ and $23 \%$ of adult patients positive for at least one organism, respectively (Figure 1A). The most prevalent organism in pediatrics detected by the FilmArray Gl Panel were Salmonella and EPEC, which were detected in $23 \%$ and $17 \%$ of pediatric patients positive for at least one organism, respectively (Figure 1B).

\section{Co-Infections}

In 16 cases, or $26.2 \%$ of positive Gl Panel results, a co-infection consisting of more than 
one organism was present. The organism most prevalent in co-infections was EPEC, which was present in $56.3 \%$ of all co-infections, followed by C. difficile and Norovirus which were both present in $37.5 \%$ of all co-infections. Only 1 EAEC infections was associated with a coinfection (Figure 2).

\section{EPEC and EAEC}

Demographics
Overall, patients that tested positive for EPEC had an average age of 33.9 years old, with $35.3 \%$ of all EPEC infections occurring in $\leq 18$ years old patients. Patients that tested positive for EAEC were an average age of 26.3 years old, with half of these occurring in $\leq 18$ year-old patients. Patients who tested positive for Norovirus were listed for comparison purposes. Patients who did not test positive for an organism on the Gl Panel were listed as No Targets Detected (NTD) (Table 2).

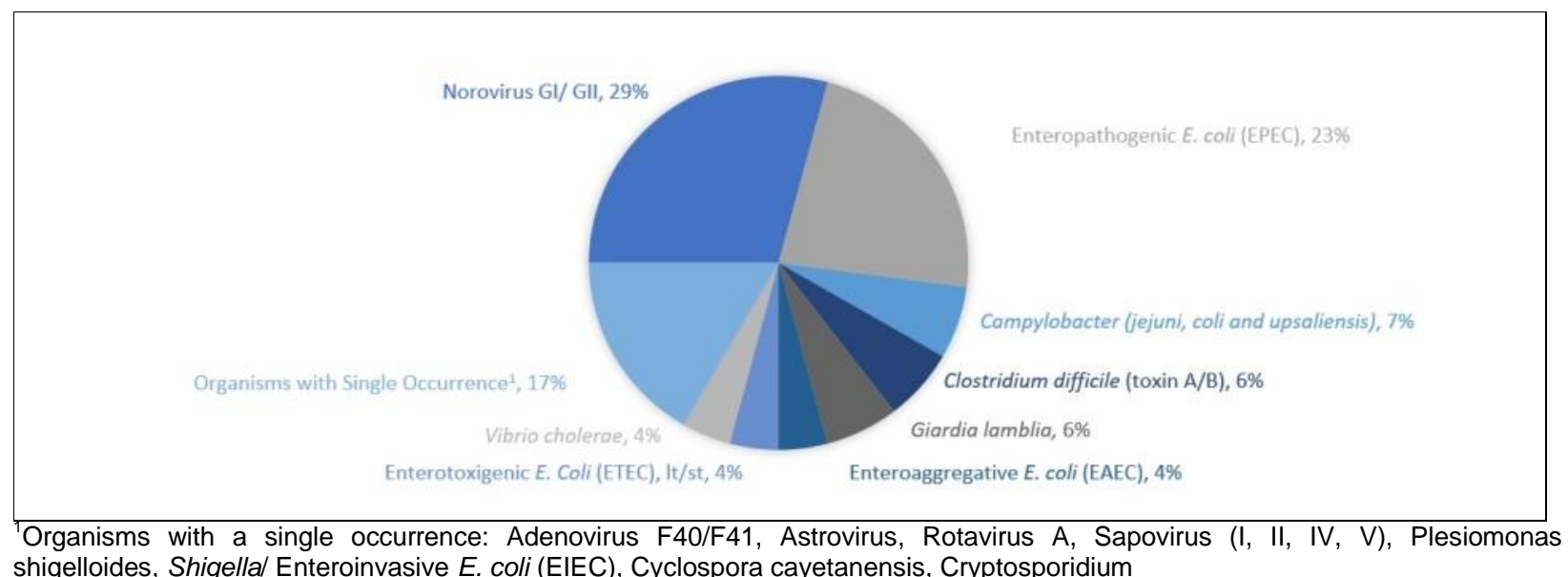

Figure 1a. Adult BioFire Positive organism breakdown.

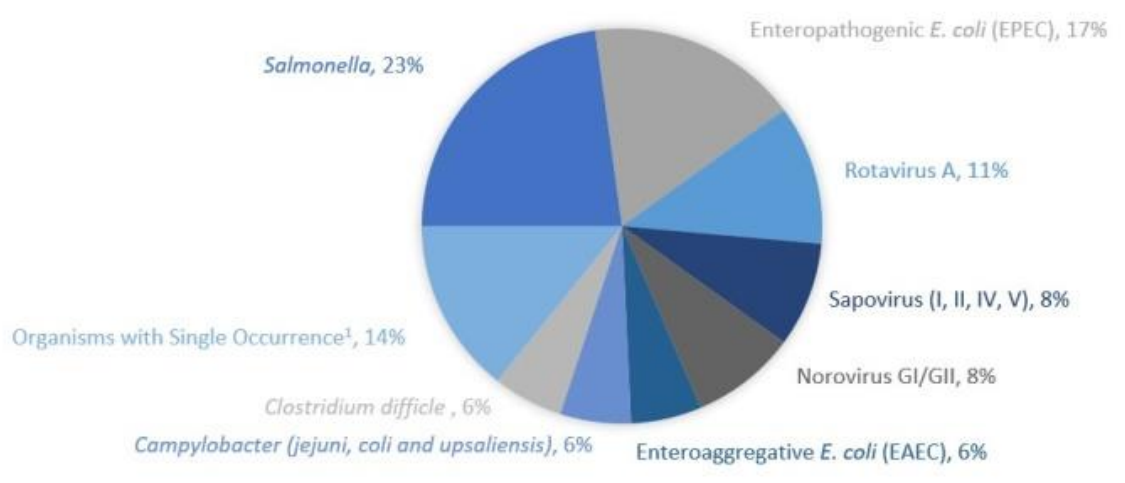

Organisms with a single occurrence: Adenovirus F40/F41, Giardia lamblia, Plesiomonas shigelloides, Shigella, Plesiomonas shigelloides, Shigella/ Enteroinvasive E. coli.

Figure 1b. Pediatric BioFire Positive Organism Breakdown 


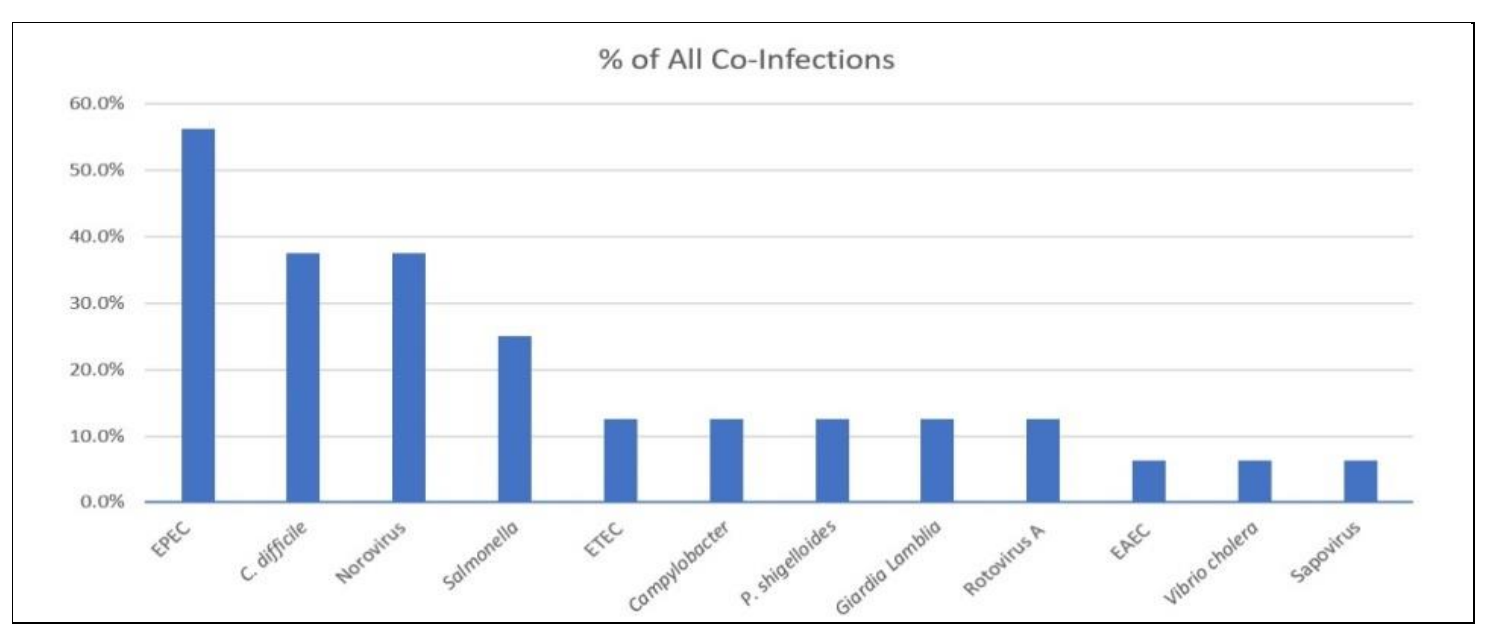

Figure 2- Percentage of Co-Infections with a specific organism.

Table 2. Patient demographics.

\begin{tabular}{|c|c|c|c|}
\hline Group/Sub-Groups (n) & Average Age & \#Patients $\leq 18$ & Male \\
\hline EPEC (17) & 33.9 & $6(35.3 \%)$ & $10(58.8 \%)$ \\
\hline No Co-Infection (8) & 31.0 & $3(37.5 \%)$ & $4(50.0 \%)$ \\
\hline Co-Infection ${ }^{1}(9)$ & 36.4 & $2(22.2 \%)$ & $6(66.7 \%)$ \\
\hline EAEC (4) & 26.3 & $2(50.0 \%)$ & $3(75.0 \%)$ \\
\hline No Co-Infection (3) & 30.3 & $1(33.3 \%)$ & $2(66.7 \%)$ \\
\hline Co-Infection ${ }^{2}(1)$ & 14.0 & $1(100 \%)$ & $1(100 \%)$ \\
\hline Norovirus (17) & 41.7 & $3(17.6 \%)$ & 7 (41.2\%) \\
\hline No Co-Infection (10) & 44.4 & $1(10.0 \%)$ & $3(30.0 \%)$ \\
\hline Co-Infection ${ }^{3}(7)$ & 37.9 & $2(28.6 \%)$ & $4(57.1 \%)$ \\
\hline NTD & 47.7 & $24(14.9 \%)$ & $78(48.4)$ \\
\hline
\end{tabular}

EPEC=Enteropathogenic E. coli, EAEC=Enteroaggregative E. coli, NTD = No targets detected

${ }^{1}$ EPEC Co-Infections involved EPEC with Clostridium difficile $(n=3)$, Salmonella $(n=3)$, Norovirus $(n=2)$, Enterotoxigenic E. coli (ETEC) $(n=2)$, Plesiomonas shigelloides $(n=1)$, Vibrio cholerae $(n=1)$, Sapovirus $(n=1)$.

${ }^{2}$ EAEC Co-Infections involved EAEC with Plesiomonas shigelloides $(\mathrm{n}=1)$.

${ }^{3}$ Norovirus Co-Infections involved Norovirus with $\operatorname{EPEC~}(n=2)$, Clostridium difficle $(n=2)$, Giardia lamblia $(n=2)$, Campylobacter $(n=1)$

Table 3. Number of Patients with specific symptoms.

\begin{tabular}{lccccccc}
\hline Group/Sub-Group (n) & $\begin{array}{c}\text { Diarrhea, } \mathbf{n} \\
(\%)\end{array}$ & $\mathbf{P} \begin{array}{c}\text { Fever }^{1}, \\
\mathbf{n}(\%)\end{array}$ & $\mathbf{p}$ & Abdominal Pain, n (\%) & $\mathbf{p}$ & $\begin{array}{c}\text { Vomiting, } \\
\mathbf{n}(\%)\end{array}$ & $\mathbf{p}$ \\
\hline EPEC (17) & $11(64.7)$ & $0.31784(23.5)$ & 0.9927 & $12(70.6)$ & $0.0425^{*}$ & $11(64.7)$ & $0.0045^{*}$ \\
No Co-Infection (8) & $6(75.0)$ & $0.95901(12.5)$ & 0.4683 & $5(62.5)$ & 0.3252 & $5(62.5)$ & 0.0580 \\
Co-Infection (9) & $5(55.6)$ & $0.17603(33.3)$ & 0.5092 & $7(77.8)$ & 0.0534 & $6(66.7)$ & $0.0239^{*}$ \\
\hline EAEC (4) & $2(50.0)$ & $0.23952(50.0)$ & 0.2250 & $1(25.0)$ & 0.4346 & $1(25.0)$ & 0.8169 \\
No Co-Infection (3) & $2(66.7)$ & $0.71691(33.3)$ & 0.6967 & $0(0)$ & 0.1233 & $1(33.3)$ & 0.9141 \\
Co-Infection (1) & $0(0)$ & $0.08051(100)$ & 0.0757 & $1(100)$ & 0.2693 & $0(0)$ & 0.5106 \\
\hline Norovirus (17) & $16(94.1)$ & $0.08641(5.9)$ & 0.0943 & $8(47.1)$ & 0.8504 & $9(52.9)$ & 0.0604 \\
No Co-Infection (10) & $9(90.0)$ & $0.30460(0)$ & 0.0824 & $4(40.0)$ & 0.7723 & $6(60.0)$ & 0.0525 \\
Co-Infection (7) & $7(100)$ & $0.13871(14)$ & 0.5570 & $4(57.1)$ & 0.5200 & $3(42.9)$ & 0.4849 \\
\hline NTD (161) & $122(75.8)$ & \multicolumn{3}{c}{$38(23.6)$} & $72(44.7)$ & & $49(30.4)$ \\
\hline
\end{tabular}

$\mathrm{NTD}=$ No targets detected, 'If reported by health care professionals. 
Table 4. Laboratory results.

\begin{tabular}{lccccc}
\hline & \multicolumn{2}{c}{ Lab Tests } & \multicolumn{3}{c}{ Lactoferrin } \\
Group/ Sub-Group & Creatinine Max & WBC Max & Positive, n (\%) & Negative & Not performed \\
\hline EPEC (17) & 0.89 & 9.6 & $4(100 \%)$ & - & 13 \\
No Co-Infection (8) & 0.96 & 8.3 & $2(100 \%)$ & - & 6 \\
Co-Infection (n=9) & 0.83 & 11.0 & $2(100 \%)$ & - & 7 \\
EAEC (4) & 0.82 & 6.2 & - & - & 4 \\
No Co-Infection (3) & 0.84 & 5.9 & - & - & 3 \\
Co-Infection (1) & 0.77 & 7.1 & - & - & 1 \\
Norovirus (17) & 1.19 & 9.54 & $1(100 \%)$ & - & 16 \\
No Co-Infection (10) & 1.43 & 9.64 & $1(100 \%)$ & - & 9 \\
Co-Infection (7) & 0.86 & 9.37 & - & - & 7 \\
NTD (161) & 1.33 & 8.17 & $24(69 \%)$ & $11(31 \%)$ & 117 \\
\hline
\end{tabular}

${ }^{1}$ Creatinine and WBC Max was the maximum value $\leq 3$ days before date of FilmArray.

Table 5. Antibiotic usage.

\begin{tabular}{lcccccc}
\hline & Amaging & Abx Given & \multicolumn{3}{c}{ ABX Management Post-GI Panel } \\
Group/Sub-Group & $\begin{array}{c}\text { Abx Given for } \\
\text { Studies }\end{array}$ & $\begin{array}{c}\text { Before }^{2} \\
\text { Gl Symptoms }\end{array}$ & Stopped $^{3}$ & Changed $^{\mathbf{4}}$ & Continued $^{4}$ \\
\hline EPEC & 0.59 & $1(6 \%)$ & $7(41 \%)$ & $4(57 \%)$ & $2(29 \%)$ & $1(14 \%)$ \\
No Co-Infection & 0.5 & $1(13 \%)$ & $3(38 \%)$ & $2(67 \%)$ & $1(33 \%)$ & $0(0 \%)$ \\
Co-Infection & 0.56 & $0(0 \%)$ & $4(44 \%)$ & $2(50 \%)$ & $1(25 \%)$ & $1(25 \%)$ \\
\hline EAEC & 0.75 & $2(50 \%)$ & $2(50 \%)$ & $0(0 \%)$ & $1(50 \%)$ & $1(50 \%)$ \\
No Co-Infection & 0.67 & $2(67 \%)$ & $2(67 \%)$ & $0(0 \%)$ & $1(50 \%)$ & $1(50 \%)$ \\
Co-Infection & 1 & $0(0 \%)$ & $0(0 \%)$ & - & - & - \\
\hline Norovirus & 0.47 & $1(6 \%)$ & $7(41 \%)$ & $2(29 \%)$ & $2(29 \%)$ & $3(42 \%)$ \\
No Co-Infection & 0.58 & $1(10 \%)$ & 2 & $1(50 \%)$ & $0(0 \%)$ & $1(50 \%)$ \\
Co-Infection & 0.14 & $0(0 \%)$ & 5 & 1 & 2 & $2(40 \%)$ \\
\hline NTD & 0.57 & $51(31 \%)$ & $63(39 \%)$ & $15(24 \%)$ & $11(17 \%)$ & $37(59 \%)$
\end{tabular}

'Average Imaging Studies conducted was the number of colonoscopies, flexible sigmoidoscopies, abdominal ultrasounds, abdominal CT Scans, or GI biopsies performed per patient \pm 3 days of the FilmArray

${ }_{2}^{2}$ Antibiotics were given $\leq 14$ days before the development of $\mathrm{Gl}$ symptoms

${ }^{3}$ Antibiotics were stopped after FilmArray GI Panel result

${ }^{4}$ Antibiotics were changed to a different antibiotic after GI Panel result

\section{DISCUSSION}

The present study investigated patient demographics, symptoms, diagnostic tests, and other characteristics through a retrospective review of medical records from patients who had stool samples tested with the BioFire FilmArray GI Panel. The aim was to ascertain the role Enteropathogenic and Enteroaggregative E. coli play in acute infectious diarrhea. It is our hope that further understanding these diseases will improve our ability to manage the care of these patients.
This chart review showed that patients who tested positive for EPEC and EAEC were often younger, with an average age of 33.9 and 26.3 years, compared to patients who did not test positive for an organism and underwent GI Panel testing (average age of 47.7). The relatively high distribution of these organisms in patients under 18 years old corresponds to findings in previous work $[9,13,25]$. We understand that age is only one aspect of the demographical characteristics important to gut microbiota. Various studies have shown that demographics such as race, substance use, 
diet, economics, and social factors may play a role in the diversity of gut microbiota [35-38]. In future studies, it will be important to analyze these various factors and the role they serve in EPEC and EAEC infections.

Overall, 61 of the $222(27.5 \%)$ samples tested on the GI Panel were positive for at least one organism. This value is lower than the overall positivity rate seen in previous studies $[9,39]$. One reason for this was that the $\mathrm{C}$. difficile result was suppressed and not included in this overall positivity rate. Patients who underwent concurrent stand-alone PCR testing specifically for $\mathrm{C}$. difficile were noted of having a co-infection if they tested positive for another organism, however, these patients were excluded if they had no other targets detected (i.e. they were not counted in the no targets detected group).

The distribution of GI Panel organisms detected was similar to those found in multi-center studies with EPEC, Campylobacter, C. difficile, Norovirus, Salmonella, and Rotavirus A being some common organisms detected throughout. Of note, only 4 patients in our study were positive for an EAEC infection while EAEC was the $3^{\text {rd }}$ and $4^{\text {th }}$ most common organism detected in these multi-center studies. The low number of positive EAEC specimens made analysis of the role EAEC played in diarrhea infections a challenge. In fact, further analysis of EAEC infections in this studied showed that only 2 had diarrhea associated with it and only 1 of these patients had only developed diarrhea after recent antibiotic use. A future study examining a larger and more diverse patient pool would almost certainly allow for better analysis of patients with EAEC infections.

We found $26.2 \%$ of all positive specimens in this study to be positive for more than one organism (co-infection), slightly lower than the rate of coinfections in multi-center studies $(30.2 \%$ in Spina et al 2015 and $32.9 \%$ in Buss et al 2015) but similar to findings in Khare et al 2014 (27.0\%) $[9,39,40]$. Similar to prior studies, we too did observe EPEC to be the most frequent pathogen detected in co-infections, making up $56.3 \%$ of all co-infections. With $52.9 \%$ of EPEC infections associated with a co-infection, one can see why the role of EPEC is being questioned.

We found that EPEC patients had a high prevalence of abdominal pain and vomiting, but not diarrhea, when compared to the presence of these symptoms in patients with no targets detected. There was no significant difference in incidence of fever and diarrhea between patients with EPEC and no targets detected. Because only four patients in this study were positive for EAEC, no significant findings could be determined, although there was a trend of less overall symptoms in these patients. As mentioned previously, of the two patients with EAEC infections that experienced diarrhea, one only developed diarrhea after antibiotic usage, so it is unlikely that development of diarrhea is reliant on an insult to the gastrointestinal flora.

All four EPEC positive samples that were also tested with lactoferrin were positive whereas only $69 \%$ of 24 infections with no targets detected tested positive for lactoferrin. Examining the two EPEC patients who tested positive with lactoferrin and had no co-infection showed that both experienced diarrhea and vomiting, with one experiencing abdominal pain as well. Prior studies by Mercado et al found that fecal leukocytes were found in $8.3 \%$ of EPEC infections and were significantly associated with diarrheal infections. Mercado et al also demonstrated that analyzing the presence of fecal lactoferrin was a sensitive method to detect inflammatory processes, detecting $95 \%$ of all diarrheagenic E. coli [41]. With all 4 of the EPEC infections experiencing some sort of symptom, providers in this study may have ordered a lactoferrin assessment in the more severe EPEC cases, thus yielding the $100 \%$ positivity rate for lactoferrin. Since $69 \%$ of patients with no targets detected on the GI Panel had a positive lactoferrin and 0 patients who had any target detected were negative for lactoferrin, the practice of using the lactoferrin test to differentiate etiologies should be questioned. Yielding a positive predictive value of $17.24 \%$ and a negative predictive value of $100 \%$, the lactoferrin test could be useful in ruling out the presence of pathogens with a negative result. However, use of a reflex testing algorithm (starting with lactoferrin and proceeding to a multiplex PCR panel) would delay results and decrease the benefits of rapid testing. Our study only tested a limited number of patients with viral pathogens, which are more likely to be negative by lactoferrin, and therefore would be missed with a reflex algorithm. 
There was no significant difference between the amounts of imaging studies ordered for patients positive for the different organisms. Additionally, there was no significant difference in how antibiotics were given for $\mathrm{Gl}$ symptoms. We can interpret this information as these infections being similar in presentation to heath care providers and thus being handled in a similar manner.

As the case with other retrospective chart reviews, this study has numerous limitations that are important to note. Firstly, it is evident that we had a low number of positive samples for numerous organisms, making analysis of symptoms for organisms like EAEC inconclusive. Additionally, $26.2 \%$ of the patients who tested positive for at least one pathogen had a co-infection, further complicating the analysis of the role of EPEC as a lone pathogen. The study also strongly relied on the reported data in patient electronic medical records, and while the same search patterns were applied to all patients in this study, inadequate history or documentation by could certainly affect results, particularly in organisms with a low number of samples. Despite these limitations, this study provides us more information on the role EPEC plays in diarrhea. We feel that the results presented in this study can be expanded upon, and with a larger sample size spanning a more diverse population, would allow for a better determination of the role EPEC and EAEC play.

\section{ACKNOWLEDGMENTS}

Declaration of Conflicting Interests: The authors declare that they have no conflict of interest.

Funding source: Research reported in this publication was supported by the University of Florida Clinical and Translational Science Institute, which is supported in part by the NIH National Center for Advancing Translational Sciences under award number UL1TR001427. The content is solely the responsibility of the authors and does not necessarily represent the official views of the National Institutes of Health.

\section{REFERENCES}

1. DuPont HL. Persistent Diarrhea: A Clinical Review. JAMA 2016 Jun 28; 315(24):2712.
2. Riddle MS, DuPont, Herbert L., Connor, Bradley A. ACG Clinical Guideline: Diagnosis, Treatment, and Prevention of Acute Diarrheal Infections in Adults. Am J Gastroenterol 2016; 111.

3. World Health Organization. World Health Organization: Diarrhoeal disease. 2016 Dec 20. http://www.who.int/mediacentre/factsheets/fs330/en/

4. Dickinson B, Surawicz CM. Infectious Diarrhea: An Overview. Curr Gastroenterol Rep 2014; 2016 16(8):399.

5. Huang JY. Infection with Pathogens Transmitted Commonly Through Food and the Effect of Increasing Use of Culture-Independent Diagnostic Tests on Surveillance-Foodborne Diseases Active Surveillance Network, 10 US Sites, 2012-2015. MMWR Morb Mortal Wkly Rep 2016; 65(14):368371.

6. Liu J, Gratz J, Maro A, et al. Simultaneous Detection of Six Diarrhea-Causing Bacterial Pathogens with an In-House PCR-Luminex Assay. J Clin Microbiol 2012; 50(1):98-103.

7. Guerrant RL, Gilder TV, Steiner TS, et al. Practice Guidelines for the Management of Infectious Diarrhea. Clin Infect Dis 2001; 32(3):331-351.

8. Barr W, Smith A. Acute diarrhea. Am Fam Physician 2014; 89(3):180-189.

9. Buss SN, Leber A, Chapin K, et al. Multicenter Evaluation of the BioFire FilmArray Gastrointestinal Panel for Etiologic Diagnosis of Infectious Gastroenteritis. J Clin Microbiol 2015; 53(3):915925.

10. Piralla A, Lunghi $G$, Ardissino $G$, et al. FilmArrayTM Gl panel performance for the diagnosis of acute gastroenteritis or hemorragic diarrhea. BMC Microbiol 2017: 17(1): 111.

11. Arenas-Hernández MMP, Martínez-Laguna $\mathrm{Y}$, Torres AG. Clinical Implications of Enteroadherent Escherichia coli. Curr Gastroenterol Rep 2012; 14(5):386-394.

12. Law RJ, Gur-Arie L, Rosenshine I, Finlay BB. In Vitro and In Vivo Model Systems for Studying Enteropathogenic Escherichia coli Infections. Cold Spring Harb Perspect Med 2013; 3(3):a009977.

13. Estrada-Garcia T, Navarro-Garcia F. Enteroaggregative Escherichia coli pathotype: a genetically heterogeneous emerging foodborne enteropathogen. FEMS Immunol Med Microbiol. 2012; 66(3):281-298.

14. Nataro JP, Kaper JB. Diarrheagenic Escherichia coli. Clin Microbiol Rev 1998; 11(1):142-201.

15. Okhuysen PC, DuPont HL. Enteroaggregative Escherichia coli (EAEC): A Cause of Acute and Persistent Diarrhea of Worldwide Importance. J Infect Dis 2010; 15; 202(4):503-505. 
16. Huppertz HI, Rutkowski S, Aleksic S, Karch H. Acute and chronic diarrhoea and abdominal colic associated with enteroaggregative Escherichia coli in young children living in Western Europe. The Lancet 1997; 349(9066):1660-1662.

17. Shin J, Oh S-S, Oh K-H, et al. An Outbreak of Foodborne Illness Caused by Enteroaggregative Escherichia coli in a High School in South Korea. Jpn J Infect Dis 2015; 68(6):514-519.

18. Hebbelstrup Jensen B, Olsen KEP, Struve C, Krogfelt KA, Petersen AM. Epidemiology and Clinical Manifestations of Enteroaggregative Escherichia coli. Clin Microbiol Rev. 2014; 27(3):614-630.

19. Aslani MM, Alikhani MY, Zavari A, Yousefi R, Zamani AR. Characterization of enteroaggregative Escherichia coli (EAEC) clinical isolates and their antibiotic resistance pattern. Int $\mathrm{J}$ Infect Dis 2011; 15(2):e136-e139.

20. Nataro JP, Yikang D, Yingkang D, Walker K. AggR, a transcriptional activator of aggregative adherence fimbria I expression in enteroaggregative Escherichia coli. J Bacteriol 1994; 176(15):46914699 .

21. Hu J, Torres AG. Enteropathogenic Escherichia coli: foe or innocent bystander? Clin Microbiol Infect 2015; $21(8): 729-734$.

22. Goosney DL, Gryenheid S, Finlay BB. Gut Feelings: Enteropathogenic $E$. coli (EPEC) Interactions with the host. Annu Rev Cell Dev Biol 2000; 16:173-189.

23. Trabulsi LR, Keller R, Gomes TAT. Typical and atypical Enteropathogenic Escherichia coli. (Synopsis). Emerg Infect Dis 2002; 8(5):508-514.

24. Ochoa TJ, Barletta F, Contreras C, Mercado E. New insights into the epidemiology of enteropathogenic Escherichia coli infection. Trans $\mathrm{R}$ Soc Trop Med Hyg 2008; 102(9):852-856.

25. Ochoa TJ, Contreras CA. Enteropathogenic Escherichia coli infection in children: Curr Opin Infect Dis 2011; 24(5):478-483.

26. Afset JE. Association of atypical enteropathogenic Escherichia coli (EPEC) with prolonged diarrhoea. J Med Microbiol 2004; 53(11):1137-1144.

27. Hernandes RT, Elias WP, Vieira MAM, Gomes TAT. An overview of atypical enteropathogenic Escherichia coli. FEMS Microbiol Lett 2009; 297(2):137-149.

28. Knutton S, Shaw R, Phillips AD, et al. Phenotypic and genetic analysis of diarrhea-associated Escherichia coli isolated from children in the United Kingdom. J Pediatr Gastroenterol Nutr 2001; 33(1):32-40.

29. Araujo JM, Tabarelli GF, Aranda KRS, et al. Typical Enteroaggregative and Atypical Enteropathogenic Types of Escherichia coli Are the
Most Prevalent Diarrhea-Associated Pathotypes among Brazilian Children. J Clin Microbiol 2007; 45(10):3396-3399.

30. Yatsuyanagi J, Saito S, Sato H, Miyajima $Y$, Amano K-I, Enomoto K. Characterization of Enteropathogenic and Enteroaggregative Escherichia coli Isolated from Diarrheal Outbreaks. J Clin Microbiol. 2002; 40(1):294-297.

31. Dulguer MV, Fabbricotti SH, Bando SY, MoreiraFilho CA, Fagundes-Neto U, Scaletsky IC. Atypical Enteropathogenic Escherichia coli Strains: Phenotypic and Genetic Profiling Reveals Strong Association between Enteroaggregative E. coli Heat Stable Enterotoxin and Dlarrhea. J Infect Dis. 2003; 188:1685-1694.

32. Harris PA, Taylor R, Thielke R, Payne J, Gonzalez $\mathrm{N}$, Conde JG. Research electronic data capture (REDCap) -- A metadata-driven methodology and workflow process for providing translational research informatics support. J Biomed Inform 2009; 42(2):377-381.

33. Campbell I. Chi-squared and Fisher-Irwin tests of two-by-two tables with small sample recommendations. Stat Med 2007; 30; 26(19):36613675.

34. Richardson JTE. The analysis of $2 \times 2$ contingency tables-yet again. Stat Med. 2011; 15; 30(8):890; author reply 891-892.

35. Chen J, Ryu E, Hathcock M, et al. Impact of demographics on human gut microbial diversity in a US Midwest population. Peer J 2016; 4:e1514.

36. Brooks AW, Priya S, Blekhman R, Bordenstein SR. Gut microbiota diversity across ethnicities in the United States. PLoS Biol 2018; 16(12):e2006842.

37. Davis SC, Yadav JS, Barrow SD, Robertson BK. Gut microbiome diversity influenced more by the Westernized dietary regime than the body mass index as assessed using effect size statistic. Microbiology Open 2017; 6(4):e00476.

38. Wen L, Duffy A. Factors Influencing the Gut Microbiota, Inflammation, and Type 2 Diabetes. J Nutr 2017; 147(7):1468S-1475S.

39. Spina A, Kerr KG, Cormican M, et al. Spectrum of enteropathogens detected by the FilmArray GI Panel in a multicentre study of community-acquired gastroenteritis. Clin Microbiol Infect 2015; $21(8): 719-728$.

40. Khare R, Espy MJ, Cebelinski E, et al. Comparative Evaluation of Two Commercial Multiplex Panels for Detection of Gastrointestinal Pathogens by Use of Clinical Stool Specimens. J Clin Microbiol 2014; 52(10):3667-3673.

41. Mercado EH, Ochoa TJ, Ecker L, et al. Fecal Leukocytes in Children Infected with Diarrheagenic Escherichia coli. J Clin Microbiol 2011; 49(4):13761381. 